\title{
Nicotinic acid/laropiprant reduces platelet count but increases mean platelet volume in patients with primary dyslipidemia
}

\author{
Anastazia Kei, Moses Elisaf
}

Department of Internal Medicine, School of Medicine, University of Ioannina, loannina, Greece

Submitted: 8 August 2011

Accepted: 25 September 2011

Arch Med Sci 2014; 10, 3: 439-444

DOI: $10.5114 /$ aoms.2014.43738

Copyright @ 2014 Termedia \& Banach

\section{Abstract}

Introduction: Nicotinic acid (NA) has been associated with reduced cardiovascular morbidity and mortality. Of note, beyond its lipid-modifying actions, NA possesses a number of not yet thoroughly defined pleiotropic actions including anti-inflammatory and antithrombotic effects. As a growing body of evidence points towards mean platelet volume (MPV) and platelet distribution width (PDW) as independent risk factors for cardiovascular disease, it would be interesting to evaluate the effect of NA on these platelet indices. Material and methods: We recruited 50 consecutive patients with dyslipidemia who were treated with a conventional statin dose $(10-40 \mathrm{mg} \mathrm{sim}$ vastatin or $10-20 \mathrm{mg}$ atorvastatin or $5-20 \mathrm{mg}$ rosuvastatin) and had not achieved the low-density lipoprotein cholesterol (LDL-C) or non-high-density lipoprotein cholesterol (non-HDL-C) goal. Add-on-statin treatment with extended release (ER) NA/laropiprant $(1,000 / 20 \mathrm{mg} /$ day for the first 4 weeks followed by $2,000 / 40 \mathrm{mg} /$ day for the next 8 weeks) was given to all patients for 3 months.

Results: The ER-NA/laropiprant resulted in a $20 \%$ reduction in platelet count

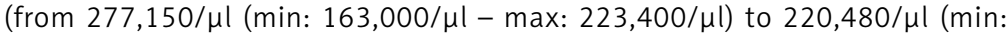
$141,000 / \mu \mathrm{l}-\max : 319,000 / \mu \mathrm{l}), p<0.001)$, while it increased MPV by $3.5 \%$ (from $11.4 \mathrm{fl}(\min : 9.2 \mathrm{fl}-\max : 13.6 \mathrm{fl})$ to $11.8 \mathrm{fl}(\min : 9.5 \mathrm{fl}-\max : 14.1 \mathrm{fl})$, $p=0.01$ ), without affecting PDW significantly (from $14.6 \mathrm{fl}$ (min: $10.5 \mathrm{fl}$ $\max : 19.3 \mathrm{fl})$ to $14.5 \mathrm{fl}(\min : 11 \mathrm{fl}-\max : 21.1 \mathrm{fl}), p=\mathrm{NS}$ ).

Conclusions: The NA is associated with reduced platelet count but with increased MPV, thereby raising questions regarding NA's antithrombotic and vasculoprotective properties.

Key words: nicotinic acid, niacin, mean platelet volume, platelets, platelet distribution width.

\section{Introduction}

A growing body of evidence points towards platelet indices as independent risk factors for cardiovascular disease. Increased mean platelet volume (MPV) has been associated with a higher rate of myocardial infarction, stroke and vascular mortality, while both high MPV and platelet distribution width (PDW) have been associated with microvascular complications in diabetic patients [1-3].

Nicotinic acid (NA) comprises the oldest hypolipidemic drug in use since 1955 [4]. A number of clinical trials have demonstrated that NA

\author{
Corresponding author: \\ Prof. Moses S. Elisaf MD, \\ FRSH, FASA, FISA \\ Department \\ of Internal Medicine \\ School of Medicine \\ University of loannina \\ 45110 loannina, Greece \\ Phone: +302651007509 \\ Fax: +302651007016 \\ E-mail: egepi@cc.uoi.gr
}


reduces cardiovascular events and total mortality in patients with coronary heart disease $[5,6]$. In addition, NA has several pleiotropic effects including anti-inflammatory, antithrombotic and anti-oxidant actions, as well as neuroprotection after stroke [7]. Of note, NA is contraindicated in patients with active arterial bleeding, since it has been associated with small reductions in platelet count (by 11\% with $2000 \mathrm{mg} /$ day extended release (ER) NA) [8]. Recently, the European Medicine Agency approved a fixed combination of extended release (ER) NA with laropiprant (a prostaglandin D2 receptor 1 (DP1) antagonist) which reduces NA-induced flushing without altering the beneficial effects of NA on lipid profile [9].

We aimed to study the effect of ER-NA/laropiprant on platelet indices in patients with primary dyslipidemia who were currently being treated with a conventional statin regimen but had not achieved treatment goals. To the best of our knowledge such a study has not been performed before. Secondary endpoints included changes in lipid profile, high sensitivity C-reactive protein (hs(RP), fasting plasma glucose, homeostasis model assessment of insulin resistance (HOMA-IR) and blood pressure (BP).

\section{Material and methods}

\section{Study population}

Consecutive subjects with primary hypercholesterolemia $(n=50)$ attending the Outpatient Lipid and Obesity Clinic of the University Hospital of Ioannina, Ioannina, Greece were recruited. Eligible patients were those treated for at least 3 months with a conventional statin dose (10$0 \mathrm{mg}$ simvastatin or 10-20 mg atorvastatin or 5$20 \mathrm{mg}$ rosuvastatin) and their low-density lipoprotein cholesterol (LDL-C) or non high-density lipoprotein cholesterol (non-HDL-C) levels were above those recommended by the National Cholesterol Education Program Adult Treatment Panel III (NCEPATP III) based on each patient's risk factors [10].

Subjects with triglycerides (TG) $>500 \mathrm{mg} / \mathrm{dl}$ $(5.65 \mathrm{mmol} / \mathrm{l})$, renal disease (serum creatinine levels $>1.6 \mathrm{mg} / \mathrm{dl} ; 141 \mu \mathrm{mol} / \mathrm{l}$ ), hypothyroidism (thyroid stimulating hormone $(\mathrm{TSH})>5 \mathrm{IU} / \mathrm{ml}$ ) and liver disease (alanine aminotransferase (ALT) and/or aspartate aminotransferase (AST) levels $>3$ times the upper limit of normal in 2 consecutive measurements) were excluded from the study. Patients with hypertension and/or diabetes were considered eligible if they were on stable medication for at least 3 months and their BP and/or glycemic profile were adequately controlled (no change in their treatment was allowed during the study period). No changes in antiplatelet, antihypertensive or antidiabetic treatment were allowed during the study.
All patients were given add-on-current-statin treatment with ER-NA/laropiprant $(1000 / 20 \mathrm{mg} /$ day for the first 4 weeks, followed by $2000 / 40 \mathrm{mg} /$ day for the next 8 weeks) for a total of 3 months.

All patients were given similar dietary advice. Compliance with treatment and lifestyle habits were assessed by questionnaire and tablet count. All study participants gave their written informed consent prior to enrolment and the Ethics Committee of the University Hospital of Ioannina approved the study protocol.

\section{Laboratory measurements}

Visits took place at baseline and 12 weeks after the start of treatment. At each visit, blood samples for laboratory tests were obtained after a 12-h overnight fast. For all patients the same EDTA standardized blood tubes were used and all blood samples were analyzed within $2 \mathrm{~h}$ after venipuncture to avoid bias due to EDTA-induced platelet swelling. All samples were processed in an automated hematology analysis system (Sysmex X2100, Kobe Japan). Serum total cholesterol (TC), HDL-C, triglycerides (TG) and fasting plasma glucose were determined enzymatically using an Olympus AU 600 analyzer (Olympus Diagnostica $\mathrm{GmbH}$, Hamburg, Germany). The LDL-C was calculated using the Friedewald equation (provided that TG were < $350 \mathrm{mg} / \mathrm{dl}(3.95 \mathrm{mmol} / \mathrm{l})$ ). The hs-CRP was measured using the N High Sensitivity CRP assay (Dade-Behring, Marburg, Germany) with particle-enhanced immunonephelometry. The reference range of the assay is $0.175 \mathrm{mg} / \mathrm{l}$ to $55 \mathrm{mg} / \mathrm{l}$. Fasting insulin levels were measured using an AzSYM microparticle enzyme immunoassay on an AzSYM analyzer (Abbott Diagnostics, Abbott Park, Illinois). HOMA-IR was calculated as follows: fasting insulin $(\mathrm{mU} / \mathrm{l}) \times$ fasting plasma glucose $(\mathrm{mg} / \mathrm{dl}) / 405$. Additionally, at each visit, BP was measured in triplicate in the right arm after patients had rested for $10 \mathrm{~min}$ in a sitting position. Measurements were performed by trained clinicians using an electronic sphygmomanometer (WatchBP Office, Microlife WatchBP AG, Widnau, Switzerland).

\section{Statistical analysis}

The Kolmogorov-Smirnov test was used to evaluate whether each parameter followed a Gaussian distribution and logarithmic transformations were accordingly performed. Values are given as mean \pm standard deviation (SD) and median (range) for parametric and non-parametric data, respectively. The differences of study parameters between baseline and post-treatment values were evaluated by paired samples $t$-test (or Wilcoxon's rank test for non-Gaussian variables). Statistical 
significance was set at $p<0.05$. Analyses were performed using SPSS version 15.0 (SPSS Inc., Chicago, Illinois).

\section{Results}

Recruitment took place from December 2009 to October 2010, and follow-up ended in January 2011. Initially, 55 Caucasian patients were enrolled. Fifty patients ( 29 men, $58 \pm 14$ years) completed the study, since 5 participants dropped out due to flushing (baseline characteristics and medications of study participants are presented in Table I). The compliance rate was $>80 \%$ in all participants who completed the study. No changes in body weight, dietary habits (including salt intake), antihypertensive, antiplatelet or antidiabetic medications were reported during follow-up.

The ER-NA/laropiprant resulted in a $20 \%$ reduction in platelet count (from 277,150/ $\mu$ l $(163,000-223,400)$ to $220,480 / \mu l \quad(141,000-$ $319,000), p<0.001)$, while it increased MPV by $3.5 \%$ (from $11.4 \mathrm{fl}(9.2-13.6)$ to $11.8 \mathrm{fl}(9.5-14.1), p=$ 0.01 ) without affecting PDW significantly (from 14.6 $\mathrm{fl}(10.5-19.3)$ to $14.5 \mathrm{fl}(11-21.1), p=\mathrm{NS})$ (Table II).

Moreover, add-on-statin ER-NA/laropiprant significantly reduced TC, TG, LDL-C and non-HDL-C by $15 \%, 25 \%, 19 \%$ and $24 \%$, respectively, while it increased HDL-C by $13 \%$ (all $p<0.01$ ), compared with baseline. In contrast, hs-CRP levels were not altered significantly (Table II). Of note, fasting plasma glucose increased by $6 \%$ (from $105 \pm 20$ $\mathrm{mg} / \mathrm{dl}$ to $111 \pm 27 \mathrm{mg} / \mathrm{dl}, p=0.01)$ and HOMA-IR increased by $27 \%$ (from $2.2(0.5-10.8)$ to 2.8 (0.517.3), $p=0.03$ ). In addition, ER-NA/laropiprant resulted in a significant $7 \%$ reduction of systolic $\operatorname{BP}(p<0.001$ vs. baseline) and a significant $5 \%$ reduction of diastolic BP ( $p=0.009$ vs. baseline) (Table II). The observed increase in MPV values was not significantly correlated with lipid, hs-CRP, BP, platelet count, fasting plasma glucose or HOMA-IR changes (data not shown).

\section{Discussion}

To the best of our knowledge this is the first study to assess the effect of ER-NA/laropiprant on platelet size indices. We demonstrated that ER-NA/laropiprant treatment is associated with a significant decrease in platelet count, while it increases MPV without significantly altering PDW.

In general, platelet size, expressed as MPV, reflects platelet activity as larger platelets represent immature, hyperactive platelets which release more prothrombotic mediators, thereby promoting atherothrombotic processes $[11,12]$. The MPV is an emerging risk factor for overall vascular mortality and ischemic heart disease, since for MPV values > $11 \mathrm{fl}$ hazard ratios were comparable to those reported for obesity or smoking [1]. More-
Table I. Baseline characteristics and medications of study participants $(n=50)$

\begin{tabular}{|c|c|}
\hline Parameter & Results \\
\hline$N$ (females/males) & $50(21 / 29)$ \\
\hline Age, mean \pm SD [years] & $58 \pm 14$ \\
\hline Current smokers, $n(\%)$ & $20(40)$ \\
\hline Diabetes mellitus, $n(\%)$ & $10(20)$ \\
\hline Metabolic syndrome, $n(\%)$ & $24(48)$ \\
\hline Body weight, mean \pm SD [kg] & $81 \pm 10$ \\
\hline $\mathrm{BMI}$, mean $\pm \mathrm{SD}\left[\mathrm{kg} / \mathrm{m}^{2}\right]$ & $29.1 \pm 3.1$ \\
\hline Waist circumference, mean $\pm \mathrm{SD}[\mathrm{cm}]$ & $99 \pm 8$ \\
\hline $\mathrm{SBP}$, mean $\pm \mathrm{SD}[\mathrm{mm} \mathrm{Hg}]$ & $134 \pm 12$ \\
\hline $\mathrm{DBP}$, mean $\pm \mathrm{SD}[\mathrm{mm} \mathrm{Hg}]$ & $81 \pm 9$ \\
\hline Hypertensive subjects, $n(\%)$ & $18(51)$ \\
\hline $\mathrm{TC}$, mean $\pm \mathrm{SD}[\mathrm{mg} / \mathrm{dl}]$ & $202 \pm 42$ \\
\hline Triglycerides [mg/dl] & $164(141-187)$ \\
\hline $\mathrm{HDL}-\mathrm{C}$, mean $\pm \mathrm{SD}[\mathrm{mg} / \mathrm{dl}]$ & $47 \pm 11$ \\
\hline LDL-C, mean \pm SD [mg/dl] & $112 \pm 35$ \\
\hline Medications at baseline & $N(\%)$ \\
\hline Clopidogrel & $8(16)$ \\
\hline Aspirin & $15(30)$ \\
\hline$\beta$-Blockers & $15(30)$ \\
\hline НСTZ & $21(42)$ \\
\hline ACEI/ARB & $22(44)$ \\
\hline Calcium channel blockers & $20(40)$ \\
\hline Metformin & $18(36)$ \\
\hline Pioglitazone & $2(4)$ \\
\hline Sulfonylurea & $10(20)$ \\
\hline Insulin & $5(10)$ \\
\hline Atorvastatin $5-20 \mathrm{mg} /$ day & $17(34)$ \\
\hline Simvastatin $10-40 \mathrm{mg} /$ day & $18(36)$ \\
\hline Rosuvastatin 5-20 mg/day & $15(30)$ \\
\hline
\end{tabular}

$B M I$ - body mass index, SBP - systolic blood pressure, DBP diastolic blood pressure, TC - total cholesterol, HDL-C - high-density lipoprotein cholesterol, LDL-C - low-density lipoprotein cholesterol, $H C T Z$ - hydrochlorothiazide, ACEI - angiotensin-converting enzyme inhibitor, ARB - angiotensin receptor blocker. Values are expressed as mean \pm standard deviation (except for triglycerides, which are expressed as median (range))

over, increased MPV has been associated with acute coronary syndrome and increased arterial stiffness $[13,14]$. Higher MPV values obtained after the onset of symptoms in patients with acute ischemic stroke were associated with larger in- 
Table II. Clinical and laboratory parameters at baseline and 3 months post-treatment

\begin{tabular}{|c|c|c|c|}
\hline Parameter & Baseline & 3 months & $\begin{array}{l}\text { Percentage } \\
\text { change (\%) }\end{array}$ \\
\hline Platelet count [platelets $/ \mu l]$ & $277,150(163,000-223,400)$ & $220,480(141,000-319,000)$ & $-20^{\dagger+}$ \\
\hline Mean platelet volume [fl] & $11.4(9.2-13.6)$ & $11.8(9.5-14.1)$ & $+3.5^{\dagger}$ \\
\hline Platelet distribution width [fl] & $14.6(10.5-19.3)$ & $14.5(11-21.1)$ & -0.7 \\
\hline $\mathrm{TC}, \mathrm{mg} / \mathrm{dl}[\mathrm{mmol} / \mathrm{l}]$ & $202 \pm 42(5.2 \pm 1.1)$ & $171 \pm 37(4.4 \pm 1)$ & $-15^{\dagger+}$ \\
\hline Triglycerides, $\mathrm{mg} / \mathrm{dl}[\mathrm{mmol} / \mathrm{l}]$ & $\begin{array}{l}164(141-187) \\
{[1.9(1.6-2.1)]}\end{array}$ & $\begin{array}{c}123(97-148) \\
{[1.4(1.1-1.7)]}\end{array}$ & $-25^{+t}$ \\
\hline $\mathrm{HDL}-\mathrm{C}, \mathrm{mg} / \mathrm{dl}(\mathrm{mmol} / \mathrm{l})$ & $47 \pm 11(1.2 \pm 0.3)$ & $53 \pm 16(1.4 \pm 0.4)$ & $+13^{\dagger}$ \\
\hline LDL-C, mg/dl (mmol/l) & $112 \pm 35(2.9 \pm 0.9)$ & $91 \pm 34(2.4 \pm 0.9)$ & $-19^{\dagger+}$ \\
\hline Non-HDL-C, mg/dl (mmol/l) & $155 \pm 37(4.0 \pm 1)$ & $118 \pm 34(3.1 \pm 0.9)$ & $-24^{\dagger \dagger}$ \\
\hline Systolic blood pressure [mm Hg] & $134 \pm 12$ & $125 \pm 10$ & $-7^{\dagger \dagger}$ \\
\hline Diastolic blood pressure [mm Hg] & $81 \pm 9$ & $77 \pm 6$ & $-5^{\dagger}$ \\
\hline Fasting plasma glucose, mg/dl (mmol/l) & $105 \pm 20(5.8 \pm 1.1)$ & $111 \pm 27(6.2 \pm 1.5)$ & $+6^{+}$ \\
\hline HOMA-IR & $2.2(0.5-10.8)$ & $2.8(0.5-17.3)$ & $+27^{\dagger}$ \\
\hline hs-CRP [mg/l] & $2.2(0.5-5.2)$ & $2.4(0.4-6.3)$ & +9 \\
\hline
\end{tabular}

TC - total cholesterol, HDL-C - high-density lipoprotein cholesterol, LDL-C - low-density lipoprotein cholesterol, non-HDL-C - nonhigh-density lipoprotein cholesterol, hs-CRP - high-sensitivity C-reactive protein, HOMA-IR-homeostasis model assessment of insulin resistance. Values are expressed as mean \pm standard deviation [except for platelets, MPV, PDW, triglycerides, HOMA-IR, hs-CRP which are expressed as median (range)]. ${ }^{\dagger} p<0.05$ vs. baseline, ${ }^{+\dagger} p<0.001$ vs. baseline

farct volume on CT brain scans and with worse clinical outcome [3]. Additionally, among patients with atrial fibrillation, those with MPV $\geq 8.9 \mathrm{fl}$ had a significantly higher stroke rate compared with patients with MPV $<8 \mathrm{fl}[15]$. In hypertensive patients MPV was associated with proteinuria, left ventricular mass index, hs-CRP, carotid intima-media thickness and $24 \mathrm{~h}$ ambulatory blood pressure measurements [16]. Higher MPV values have also been associated with increased coronary heart disease prevalence in diabetic patients, thrombolysis failure in patients with ST-elevation myocardial infarction, as well as decompensation and death in patients with heart failure [17-19]. Data regarding the effects of drugs on MPV are few. Aspirin has no effect on MPV, while treatment with rosuvastatin (10 mg/day for 12 weeks) was associated with MPV reduction (from $8.4 \pm 1.2 \mathrm{fl}$ to $8.1 \pm 1.3 \mathrm{fl}, p<0.001$ ) in dyslipidemic patients [20, 21]. Of note, this effect was not correlated with changes in plasma lipids, as was the case in our study with ER-NA/laropiprant [21].

Similarly, PDW, which measures the variability in platelet size, represents another marker of platelet activation [22]. It has been demonstrated that PDW values are higher in patients with rheumatoid arthritis compared with healthy subjects, while among patients with rheumatoid arthritis both PDW and MPV values were found higher in those who also had cardiovascular disease [23]. In addition, both MPV and PDW were found higher in diabetic patients compared with healthy controls, while among diabetics PDW was higher in those with microvascular complications [2]. However, data are controversial since in a large prospective trial with 1882 patients with coronary artery disease undergoing coronary angiography, PDW was not associated with the prevalence of coronary artery disease or with carotid intima-media thickness [24]. Of note, it has been established that MPV associates directly with PDW [22]. However, in our study ER-NA/laropiprant had no significant effect on PDW, despite significantly increasing MPV.

Even though there are only a few data, laropiprant does not seem to affect platelet reactivity when given alone or with ER-NA $[25,26]$. On the other hand, laropiprant's effect on platelets cannot be totally excluded since laropiprant has modest activity at platelets' thromboxane receptor (TP), which may alter platelet function either by enhancing platelet reactivity through DP1 antagonism or by inhibiting platelet aggregation through TP antagonism. In fact, laropiprant has been associated with a small, transient inhibition of platelet responsiveness to collagen without clinically meaningful alteration of platelet function [26]. Moreover, concomitant administration of aspirin or clopidogrel with NA and/or laropiprant may not have the same effect on platelets compared to NA monotherapy. In fact, concomitant daily administration of laropiprant $40 \mathrm{mg}$ with clopidogrel $75 \mathrm{mg}$ or aspirin $81 \mathrm{mg}$ resulted in a $4-5 \%$ in- 
crease in bleeding time at $24 \mathrm{~h}$ after the last dose compared to bleeding time after treatment with clopidogrel or aspirin alone. On the other hand, inhibition of platelet aggregation was not affected by co-administration of laropiprant [27]. Of note, in our study almost half of the participants (45\%) were on aspirin and/or clopidogrel. The reported increase in MPV was similar in the subgroup of patients receiving compared to patients not receiving antiplatelet therapy, even though the small study population does not allow us to clarify aspirin's or clopidogrel's effect. Noteworthy, taking into consideration the fact that ER-NA/laropiprant treatment lowers BP and elevates HDL-C, it comes as a surprise that ER-NA/laropiprant did not result in MPV reduction, as both hypertension and low HDL-C levels have been directly correlated with increased MPV levels [16, 28-30]. But how did ER-NA/laropiprant increase MPV? Since MPV has been directly correlated with fasting plasma glucose levels and the HOMA index, an explanation could be that the ER-NA/laropiprant-induced increases in fasting plasma glucose levels and in insulin resistance could result in higher MPV values $[22,28]$. However, MPV changes were not significantly correlated with fasting plasma glucose and HOMA-IR changes in our study. Moreover, MPV has been occasionally inversely correlated with platelet count; thus the ER-NA/laropiprant-induced platelet count reduction may have resulted in the reported increase in MPV levels [24, 28]. However, platelet count reduction was not correlated with MPV changes in our study population.

In the Atherothrombosis Intervention in Metabolic syndrome with low $\mathrm{HDL} /$ high triglycerides: Impact on Global Health outcomes (AIM-HIGH) trial 3500 patients with established cardiovascular disease and dyslipidemia who received simvastatin with or without ezetimibe at a dose sufficient to maintain LDL-C at $40-80 \mathrm{mg} / \mathrm{dl}$ were randomized to receive ER-NA (1500-2000 mg/day) or placebo. The primary end point was time to the first occurrence of coronary heart disease death, nonfatal myocardial infarction, ischemic stroke, hospitalization for acute coronary syndrome or symptom-driven coronary or cerebral revascularization with average follow-up of 4.1 years. However, the trial was halted prematurely, 18 months ahead of schedule, as ER-NA treatment offered no additional benefit beyond statin therapy, while a small, unexplained increase in ischemic stroke rate was reported in the NA group [31, 32]. The increased stroke rate can be associated with our findings regarding the effect of ER-NA on MPV, taking into consideration that MPV may comprise an independent risk factor for stroke [3, 15]. However, the small number of patients in our study does not allow firm conclusions.
Moreover, in The Heart Protection Study 2-Treatment of HDL to Reduce the Incidence of Vascular Events (HPS-2 THRIVE) study, 25,673 patients with established vascular disease on simvastatin ( \pm ezetimibe) were randomized to ER-NA/LRPT (2000/40 mg/day) or placebo. After nearly 4 years of follow-up ER-NA/LRPT did not significantly reduce coronary deaths, nonfatal myocardial infarctions, strokes, or coronary revascularizations compared with statin ( \pm ezetimibe) monotherapy. What is more, ER-NA/LRPT was associated with an excess of serious nonfatal side effects which led to the decision to suspend this drug worldwide.

In conclusion, overall, ER-NA/laropiprant increased MPV in patients with primary dyslipidemia. This result may provide a piece of the puzzle of how the oldest hypolipidemic drug works and raises concerns about yet unknown pleiotropic actions of NA. However, whether this effect of NA on MPV translates into increased cardiovascular risk remains to be established.

\section{References}

1. Slavka G, Perkmann T, Haslacher H, et al. Mean platelet volume may represent a predictive parameter for overall vascular mortality and ischemic heart disease. Arterioscler Thromb Vasc Biol 2011; 31: 1215-8.

2. Jindal S, Gupta S, Gupta R, et al. Platelet indices in diabetes mellitus: indicators of diabetic microvascular complications. Hematology 2011; 16: 86-9.

3. Pikija S, Cvetko D, Hajduk M, Trkulja V. Higher mean platelet volume determined shortly after the symptom onset in acute ischemic stroke patients is associated with a larger infarct volume on CT brain scans and with worse clinical outcome. Clin Neurol Neurosurg 2009; 111: 568-73.

4. Carlson LA. Nicotinic acid: the broad-spectrum lipid drug. A 50th anniversary review. J Intern Med 2005; 258: 94-114.

5. Brown BG, Zhao XQ, Chait A, et al. Simvastatin and niacin, antioxidant vitamins, or the combination for the prevention of coronary disease. N Engl J Med 2001; 345 : 1583-92.

6. Canner PL, Berge KG, Wenger NK, et al. Fifteen year mortality in coronary drug project patients: long-term benefit with niacin. J Am Coll Cardiol 1986; 8: 1245-55.

7. Florentin M, Liberopoulos EN, Kei A, Mikhailidis DP, Elisaf MS. Pleiotropic effects of nicotinic acid: beyond high density lipoprotein cholesterol elevation. Curr Vasc Pharmacol 2011; 9: 385-400.

8. NIASPAN [package insert]. North Chicago, IL: Abbott Laboratories http://www.rxabbott.com/pdf/niaspan.pdfNIASPAN

9. TREDAPTIVE product overview. [Online] [cited 2008]. Available from:http://www.ema.europa.eu/humandocs/ Humans/EPAR/tredaptive/tredaptive.htm.

10. Expert Panel on Detection Evaluation And Treatment of High Blood Cholesterol In Adults. Executive Summary of The Third Report of The National Cholesterol Education Program (NCEP) Expert Panel on Detection, Evaluation, And Treatment of High Blood Cholesterol In Adults (Adult Treatment Panel III). JAMA 2001; 285: 2486-97. 
11. Thompson CB, Jakubowski JA, Quinn PG, Deykin D, Valeri CR. Platelet size and age determine platelet function independently. Blood 1984; 63: 1372-5.

12. Davi G, Patrono C. Platelet activation and atherothrombosis. N Engl J Med 2007; 357: 2482-94.

13. Wang RT, Li Y, Zhu XY, Zhang YN. Increased mean platelet volume is associated with arterial stiffness. Platelets 2011; 22: 447-51. .

14. Lippi G, Filippozzi L, Salvagno GL, et al. Increased mean platelet volume in patients with acute coronary syndromes. Arch Pathol Lab Med 2009; 133: 1441-3.

15. Ha SI, Choi DH, Ki YJ, et al. Stroke prediction using mean platelet volume in patients with atrial fibrillation. Platelets 2011; 22: 408-14.

16. Yarlioglues M, Kaya MG, Ardic I, et al. Relationship between mean platelet volume levels and subclinical target organ damage in newly diagnosed hypertensive patients. Blood Press 2011; 20: 92-7.

17. Pereg D, Berlin T, Mosseri M. Mean platelet volume on admission correlates with impaired response to thrombolysis in patients with ST-elevation myocardial infarction. Platelets 2010; 21: 117-21.

18. Kandis H, Ozhan H, Ordu S, et al. The prognostic value of mean platelet volume in decompensated heart failure. Emerg Med J 2011; 28: 575-8.

19. Tavil Y, Sen N, Yazici H, et al. Coronary heart disease is associated with mean platelet volume in type 2 diabetic patients. Platelets 2010; 21: 368-72.

20. Jagroop IA, Tsiara S, Mikhailidis DP. Mean platelet volume as an indicator of platelet activation: methodological issues. Platelets 2003; 14: 335-6.

21. Coban E, Afacan B. The effect of rosuvastatin treatment on the mean platelet volume in patients with uncontrolled primary dyslipidemia with hypolipidemic diet treatment. Platelets 2008; 19: 111-4.

22. Muscari A, De Pascalis S, Cenni A, et al. Determinants of mean platelet volume (MPV) in an elderly population relevance of body fat, blood glucose and ischaemic electrocardiographic changes. Thromb Haemost 2008; 99 1079-84.

23. Jurcut C, Jurcut R, Caraiola S, et al. Platelet histogram indices and cardiovascular disease in patients with rheumatoid arthritis. Rom J Intern Med 2010; 48: 51-5.

24. De Luca G, Venegoni L, lorio S, et al. Platelet distribution width and the extent of coronary artery disease: results from a large prospective study. Platelets 2010; 21: 508-14.

25. Lauring B, Dishy V, Luo WL, et al. Laropiprant in combination with extended-release niacin does not alter urine 11-dehydrothromboxane B2, a marker of in vivo platelet function, in healthy, hypercholesterolemic, and diabetic subjects. J Clin Pharmacol 2009; 49: 1426-35.

26. Lai E, Schwartz J, Dallob A, et al. Effects of extended release niacin/laropiprant, laropiprant, extended release niacin and placebo on platelet aggregation and bleeding time in healthy subjects. Platelets 2010; 21: 191-8.

27. Dallob A, Luo WL, Luk JM, et al. The effects of laropiprant, a selective prostaglandin $\mathrm{D}(2)$ receptor 1 antagonist, on the antiplatelet activity of clopidogrel or aspirin. Platelets 2011; 22: 495-503.

28. Kei A, Liberopoulos EN, Elisaf MS. What restricts the clinical use of nicotinic acid? Curr Vasc Pharmacol 2011; 9: 521-30.

29. Arslan N, Makay B. Mean platelet volume in obese adolescents with nonalcoholic fatty liver disease. J Pediatr Endocrinol Metab 2010; 23: 807-13.

30. Kei A, Elisaf M, Moutzouri E, Tsiara S, Liberopoulos E. Add-on-statin extended release nicotinic acid/laropip- rant but not switch to high-dose rosuvastatin lowers blood pressure: an open-label randomized study. Int J Hypertens 2011; 2011: 830434

31. AIM-HIGH investigators. The role of niacin in raising high-density lipoprotein cholesterol to reduce cardiovascular events in patients with atherosclerotic cardiovascular disease and optimally treated low-density lipoprotein cholesterol: baseline characteristics of study participants. The Atherothrombosis Intervention in $\mathrm{Me}$ tabolic syndrome with low HDL/high triglycerides: impact on Global Health outcomes (AIM-HIGH) trial. Am Heart J 2011; 161: 538-43.

32. NIH pulls plug on AIM-HIGH trial with niacin http:// www.theheart.org/article/1231453.do?utm campaign=newsletter \&utm_medium =email \&utm source=20110601 topTen mai [cited 27 May, 2011].

33. HPS2-THRIVE Collaborative Group. HPS2-THRIVE randomized placebo-controlled trial in 25673 high-risk patients of ER niacin/laropiprant: trial design, pre-specified muscle and liver outcomes, and reasons for stopping study treatment. Eur Heart J 2013; 34: 1279-91. 